\title{
Associations between Household-Level Exposures and All-Cause Diarrhea and Pathogen-Specific Enteric Infections in Children Enrolled in Five Sentinel Surveillance Studies
}

Josh M. Colston ${ }^{1}$, Abu S. G. Faruque ${ }^{2}$, M. Jahangir Hossain ${ }^{3}$, Debasish Saha ${ }^{4}$, Suman Kanungo ${ }^{5}$, Inácio Mandomando ${ }^{6}$, M. Imran Nisar ${ }^{7}{ }^{\circledR}$, Anita K. M. Zaidi ${ }^{7}$, Richard Omore ${ }^{8}$, Robert F. Breiman ${ }^{9}$, Samba O. Sow ${ }^{10}$, Anna Roose ${ }^{11}{ }^{(D}$, Myron M. Levine ${ }^{12}$, Karen L. Kotloff ${ }^{13}$, Tahmeed Ahmed ${ }^{14}$, Pascal Bessong ${ }^{15}$, Zulfiqar Bhutta ${ }^{16}$, Estomih Mduma ${ }^{17}$, Pablo Penatero Yori ${ }^{1}$, Prakash Sunder Shrestha ${ }^{18}$, Maribel P. Olortegui ${ }^{19}$, Gagandeep Kang ${ }^{20}$, Aldo A. M. Lima ${ }^{21}$, Jean Humphrey ${ }^{22}$, Andrew Prendergast ${ }^{23}$, Francesca Schiaffino ${ }^{24}(\mathbb{D}$, Benjamin F. Zaitchik ${ }^{25}$ and Margaret N. Kosek ${ }^{26, *}$

1 Division of Infectious Diseases and International Health, University of Virginia School of Medicine, Charlottesville, VA 22903, USA; josh.colston@virginia.edu (J.M.C.); pyori@virginia.edu (P.P.Y.)

2 Centre for Nutrition \& Food Security, International Centre for Diarrhoeal Disease Research, Dhaka 1212, Bangladesh; gfaruque@icddrb.org

3 Medical Research Council Unit-The Gambia at the London School of Hygiene \& Tropical Medicine, Banjul P.O. Box 273, Republic of Gambia; jhossain@mrc.gm

4 Epidemiology and Health Economics, GSK Vaccines, 1300 Wavre, Belgium; debasish.x.saha@gsk.com

5 Suman Kanungo-National Institute of Cholera and Enteric Diseases, Kolkota 700010, India; sumankanungo@gmail.com

6 Centro de Investigação em Saúde de Manhiça, Manhica CP 1929, Mozambique; inacio.mandomando@manhica.net

7 Department of Pediatrics and Child Health, The Aga Khan University, Karachi 74800, Pakistan; imran.nisar@aku.edu (M.I.N.); anita.zaidi@aku.edu (A.K.M.Z.)

8 Kenya Medical Research Institute, Center for Global Health Research, Kisumu, Nyanza 40100, Kenya; omorerichard@gmail.com

9 Hubert Department of Global Health, Rollins School of Public Health, Emory University, Atlanta, GA 30322, USA; rfbreiman@emory.edu

10 Centre pour le Développement des Vaccins, Bamako BP 251, Mali; ssow@som.umaryland.edu

11 Indiana University School of Medicine, Indianapolis, IN 46202, USA; awroose@iu.edu

12 Departments of Medicine and Pediatrics, Center for Vaccine Development and Global Health, University of Maryland School of Medicine, Baltimore, MD 21201, USA; mlevine@som.umaryland.edu

13 Department of Pediatrics, Center for Vaccine Development and Global Health, University of Maryland School of Medicine, Baltimore, MD 21201, USA; kkotloff@som.umaryland.edu

14 Nutrition and Clinical Services Division, International Centre for Diarrhoeal Disease Research, Bangladesh (icddr,b), Dhaka 1212, Bangladesh; tahmeed@icddrb.org

15 HIV/AIDS \& Global Health Research Programme, University of Venda, Thohoyandou, Limpopo 0950, South Africa; Pascal.Bessong@univen.ac.za

16 Department of Pediatrics and Child Health, Aga Khan University, Karachi 74800, Pakistan; zulfiqar.bhutta@aku.edu

17 Haydom Global Health Institute, Haydom P.O. Box 9000, Tanzania; estomduma@gmail.com

18 Department of Child Health, Institute of Medicine of Tribhuvan University, Kirtipur 44618, Nepal; prakashsunder@hotmail.com

19 Asociacion Benefica PRISMA, Iquitos 16006, Peru; mparedeso@prisma.org.pe

20 Department of Gastrointestinal Sciences, Christian Medical College, Vellore 632004, India; gkang@cmcvellore.ac.in

21 Department of Physiology and Pharmacology, Faculty of Medicine, Federal University of Ceará, Fortaleza 60020-181, Brazil; alima@ufc.br 
22 Department of International Health, Johns Hopkins Bloomberg School of Public Health, Baltimore, MA 21205, USA; jhumphr2@jhu.edu

23 Centre for Paediatrics, Blizard Institute, Queen Mary University of London, London E1 2AT, UK; a.prendergast@qmul.ac.uk

24 Faculty of Veterinary Medicine, Universidad Peruana Cayetano Heredia, Lima 15102, Peru; francesca.schiaffino@upch.pe

25 Department of Earth and Planetary Sciences, Johns Hopkins Krieger School of Arts and Sciences, Baltimore, MA 21218, USA; zaitchik@jhu.edu

26 Division of Infectious Diseases, International Health and Public Health Sciences, Department of Internal Medicine, University of Virginia, Charlottesville, VA 22903, USA

* Correspondence: mkosek@virginia.edu; Tel.:+1-434-982-6768

Received: 27 August 2020; Accepted: 25 September 2020; Published: 2 November 2020

Abstract: Diarrheal disease remains a major cause of childhood mortality and morbidity causing poor health and economic outcomes. In low-resource settings, young children are exposed to numerous risk factors for enteric pathogen transmission within their dwellings, though the relative importance of different transmission pathways varies by pathogen species. The objective of this analysis was to model associations between five household-level risk factors-water, sanitation, flooring, caregiver education, and crowding - and infection status for endemic enteric pathogens in children in five surveillance studies. Data were combined from 22 sites in which a total of 58,000 stool samples were tested for 16 specific enteropathogens using qPCR. Risk ratios for pathogen- and taxon-specific infection status were modeled using generalized linear models along with hazard ratios for all-cause diarrhea in proportional hazard models, with the five household-level variables as primary exposures adjusting for covariates. Improved drinking water sources conferred a 17\% reduction in diarrhea risk; however, the direction of its association with particular pathogens was inconsistent. Improved sanitation was associated with a $9 \%$ reduction in diarrhea risk with protective effects across pathogen species and taxa of around 10-20\% risk reduction. A 9\% reduction in diarrhea risk was observed in subjects with covered floors, which were also associated with decreases in risk for zoonotic enteropathogens. Caregiver education and household crowding showed more modest, inconclusive results. Combining data from diverse sites, this analysis quantified associations between five household-level exposures on risk of specific enteric infections, effects which differed by pathogen species but were broadly consistent with hypothesized transmission mechanisms. Such estimates may be used within expanded water, sanitation, and hygiene (WASH) programs to target interventions to the particular pathogen profiles of individual communities and prioritize resources.

Keywords: enteropathogens; water; sanitation and hygiene; child health; diarrheal disease; zoonoses

\section{Introduction}

Despite considerable progress in recent years, diarrheal disease remains a major cause of mortality and morbidity in childhood [1,2] — responsible for some 446,000 under-five deaths in 2016 [3]—and its sequelae lead to poor health and economic outcomes in adulthood $[4,5]$. New diagnostic and statistical methods, as well as a number of ambitious multi-site, population-based studies have shed light on the pathogen-specific etiologies of diarrheal disease [6-8]. Numerous microbial agents each with distinct transmission dynamics interact with host-level, environmental, and meteorological risk factors to cause enteric infections which may be symptomatic or sub-clinical $[9,10]$. Understanding these complexities may in turn assist in the identification of tailored interventions to reduce the impact of enteric disease.

In low-resource settings, infants and young children are exposed to numerous risk factors for enteric pathogen transmission within their homes, though the relative importance of different transmission pathways vary depending on the pathogen type and species [11]. While food- and 
water-borne transmission are the dominant pathways for most bacteria and protozoa, enteric viruses appear to depend on more direct person-to-person contact [12], an observation supported by high rates of infection even in settings with developed water and sanitation infrastructures. Inadequate sanitation and drinking water are leading household-level risk factors for all-cause childhood diarrhea but, while systematic reviews have estimated that improvements to these facilities can reduce the risk of this outcome by between $25 \%$ and $75 \%[13,14]$, three high-profile randomized control trials found more mixed, context-dependent effects [15]. Furthermore, they likely do not impact transmission of rotavirus [16], one of the pathogens responsible for the largest share of the diarrheal disease burden $[2,17,18]$. Floors that are covered with wood, tiles, or cement may protect against transmission of some pathogens compared to floors made of packed earth or sand [19-22]; however, the underlying mechanisms are unclear. The link between a mother's level of education and the health, nutrition, and mortality prospects of her offspring is credited with having averted 4.2 million child deaths between 1970 and 2009 due to increased female educational attainment [23-25] and associations between caregiver education and diarrheal disease outcomes have been documented [22,26]. Finally, household crowding, whereby the number of occupants of a dwelling exceeds the capacity of its space, is a risk factor for numerous adverse health conditions including diarrheal disease [27]. While several studies have attempted to characterize the associations underlying these risk factors by taxa-separately for viral, bacterial, and protozoal agents [22,28] — pathogen species-specific analyses have, until very recently, not been possible due to the prohibitive sample sizes and limitation in diagnostic capacity using culture based methods or targeted PCR for fewer than five pathogens concurrently [29].

The objective of the analysis reported here was to model the associations between five household-level risk factors-water, sanitation, floor material, caregiver education, and household crowding-and infection status for 16 common, high-burden enteric pathogens ascertained in children aged 0-5 years enrolled in five surveillance studies. The a priori hypothesis to be tested was that improved sanitation and water sources, finished floors, caregiver primary school completion, and lack of crowding are protective against enteric infection, but to an extent that differs by pathogen species and taxon.

\section{Materials and Methods}

\subsection{Study Population}

This analysis combined data from five studies. Studies were considered eligible for inclusion if they used polymerase chain reaction (PCR) to diagnose the same panel of 16 enteropathogens, if they had collected data relating to all 5 exposures and if they were willing to share data for the purposes of the analysis. The Etiology Risk Factors and Interactions of Enteric Infections and Malnutrition and the Consequences for Child Health and Development (MAL-ED) project was a multi-site cohort study in which 2100 newborns (227-303 per site) were recruited from communities in eight different Low- and Middle-Income countries and were monitored continuously over their first 2 years of life [30]. At the MAL-ED site in Loreto, Peru, a shorter cohort study was carried out to evaluate novel biomarkers of environmental enteropathy ("Novel Biomarkers"). The Global Enteric Multicenter Study (GEMS) was a multi-site case-control study, in which cases of moderate-to-severe diarrhea (MSD) in children under 5 years old presenting at health facilities were matched by age, sex, and time of presentation of the index case with healthy controls recruited from the same community at sites in seven countries [31]. The Study of the Etiology of Childhood Diarrhea in the Brazilian Semiarid Region (part of the RECODISA-Rede de Ovino Caprino Cultura e Diarréia Infantil no Semi-Árido Brasileiro-network) was a case-control study carried out in six Brazilian cities in which cases with diarrhea and asymptomatic, age-matched controls were identified among children aged 2-36 months by community-based active surveillance [8]. The Sanitation Hygiene Infant Nutrition Efficacy (SHINE) Trial was a cluster-randomized, community-based trial in 2 rural districts of Zimbabwe that included an embedded sub-study of environmental enteric dysfunction, in which data and biospecimens were 
collected from infants longitudinally up to 18 months of age [32]. The locations of the 22 study sites are shown in Figure 1.

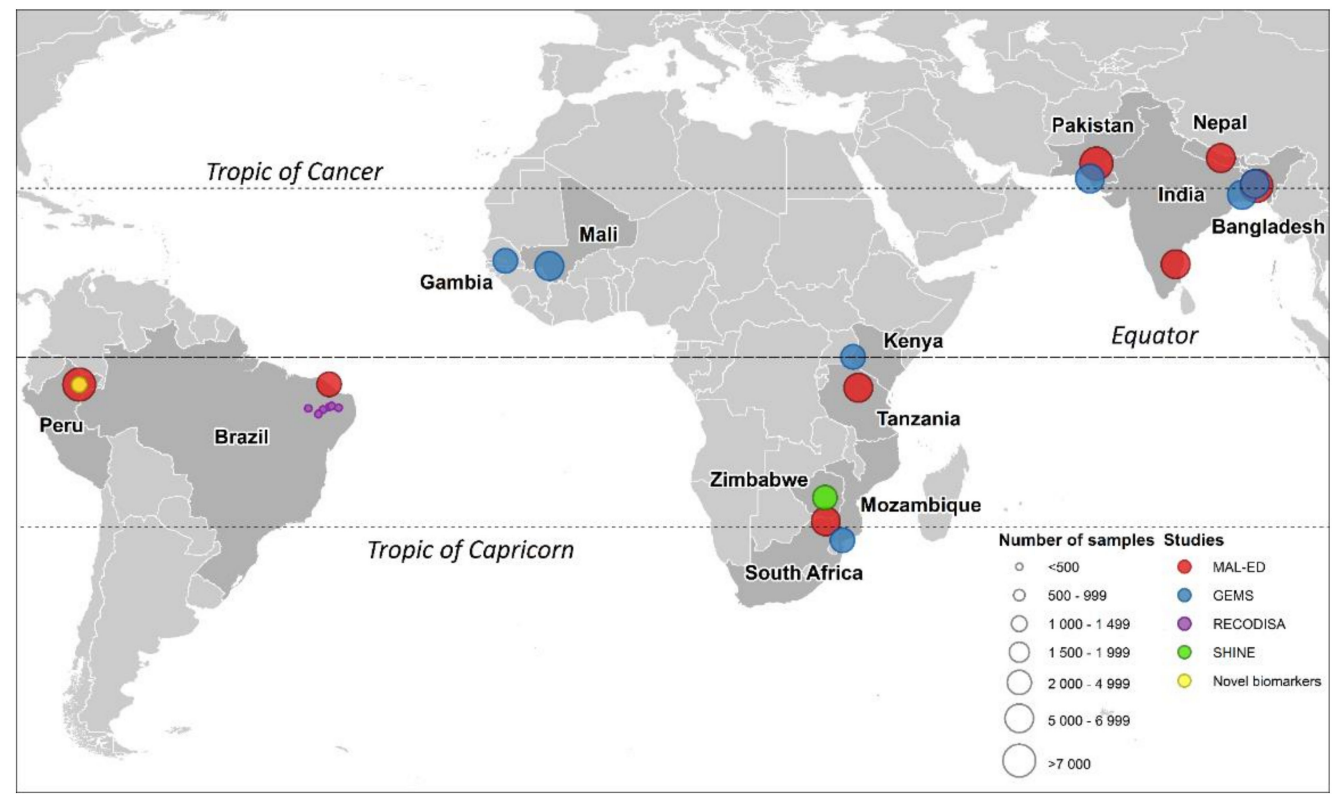

Figure 1. Locations of the 22 study sites in which the 5 studies were carried out and numbers of available stool samples (before exclusion of samples not diagnosed by qPCR).

\subsection{Outcome Variable}

In the MAL-ED, Novel Biomarkers, and SHINE studies, stool samples were collected from the subjects by locally recruited fieldworkers according to a predefined schedule (at monthly intervals following enrollment in MAL-ED, between 1 and 30 days after enrollment in Novel Biomarkers and at 1, 3, 6, and 12 months of age in SHINE) and upon reporting of a diarrheal episode by the child's caregiver. In the other studies, locally recruited fieldworkers collected a single stool sample from each case and their matched control subject upon enrollment (although in GEMS it was possible for the same individual to be enrolled more than once and therefore contribute multiple samples, but this occurred infrequently). Enteropathogen-specific infection status was ascertained using probe-based quantitative PCR (qPCR) assays on custom-developed TaqMan Array Cards (Thermo Fisher, Waltham, MA, USA) [33] for all stool samples in Novel Biomarkers, SHINE, and RECODISA and for large subsets of the GEMS and MAL-ED samples. Samples from MAL-ED and GEMS that were tested using other methods were excluded from this analysis for consistency given the differing diagnostic sensitivities (this meant that only pathogen data from GEMS1 and not GEMS1A were included in the analysis). For consistency across studies, where $\mathrm{Ct}$ values were available a cutoff value of 35 cycles was used. Infection status for each of 16 highly prevalent or endemic enteric pathogen species or pathotypes were treated as binary outcome variables as was infection status for any of the three pathogen taxa-viruses, bacteria, and protozoa. The pathogen species were: adenovirus, astrovirus, norovirus, rotavirus, sapovirus, Aeromonas, Campylobacter, enteroaggregative Escherichia coli (E. coli) (EAEC), enteropathogenic E. coli, ((EPEC) typical and atypical), heat-labile enterotoxigenic E. coli (LT-ETEC), and heat-stable ETEC (ST-ETEC), Salmonella, Shigella/enteroinvasive E. coli (EIEC) (qPCR uses the same gene target for these two closely related pathogens), Cryptosporidium, and Giardia. Several important pathogens (e.g., Vibrio, Entamoeba, helminths) were excluded from consideration due to there having been too few detections to fit the regression models, or due to not having been tested for in every study. To ensure that a single infection episode was not counted multiple times, Campylobacterand norovirus-positive samples were excluded if they were collected within 30 days of a previous sample that was positive for the same pathogen strain without being separated by an intermediate 
negative sample [9]. For all other pathogens that are less associated with persistent infection, a 14-day period was used, except for the two protozoa, for which samples that were positive for the same species (Cryptosporidium parvum or Cryptosporidium hominis) or assemblage (Giardia duodenalis A or B) as a prior sample from the same subject were excluded unless separated by three negative samples in recognition of their association with chronic infections.

\subsection{Covariates}

The primary exposures of interest were the following binary household-level factors, selected on the basis of their hypothesized association with enteric pathogen transmission and the comparability of their definitions across the contributing studies:

- Drinking water: whether or not the subject resided in a household with access to an improved drinking water source (with potential to deliver safe water by nature of its design and construction such as piped water or protected tubewells, boreholes, dug wells, or springs) [34].

- Sanitation: whether or not the subject resided in a household with access to an improved, non-shared sanitation facility ("improved" meaning designed to hygienically separate excreta from human contact) [34].

- Flooring material: whether or not the subject resided in a household that had a covered ("improved" — rudimentary or finished) as opposed to natural ("unimproved"—earth or sand) floor [35].

- Caregiver education: a binary variable indicating whether or not the subject's caregiver had completed primary education ( $\geq 6$ completed years of schooling [25]).

- Household crowding: a binary variable indicating whether or not the subject resided in a household with 3 or more residents per bedroom [22].

Definitions of these variables used by each study were mapped as closely as possible to those used in previous publications from the World Health Organization, the Demographic and Health Surveys and others, as shown in Table S2 in the supplementary material. For subjects who had multiple assessments of these variables during follow-up, the first available value was used, for consistency with those studies that only assessed them at baseline. In addition, the following covariates were included as potential confounders:

- Site: a categorical variable indicating at which of the 22 study sites the subject was enrolled, included to adjust both for between-site differences in background pathogen transmission levels and for potential confounding engendered by differences in surveillance methods between the 5 studies.

- $\quad$ Sample type: whether the stool sample was collected during a diarrheal episode (cases of diarrhea in GEMS and RECODISA, diarrheal collections in MAL-ED, Novel Biomarkers and SHINE) or while the subject was asymptomatic (controls in GEMS and RECODISA, surveillance samples in MAL-ED, Novel Biomarkers and SHINE).

- Age: the subjects' age in continuous months at the time of stool sample collection, modeled using linear, quadratic, and cubic terms to account for non-linearity of association with enteric pathogen presence.

- Feeding status: a categorical variable indicating whether the child was being exclusively breastfed, partially breastfed or had been fully weaned (no longer receiving any breastmilk) at the time of sample collection.

- Nutritional status: two binary variables indicating whether or not the child was moderately or severely stunted or underweight (respectively, a length-for-age and weight-for-age Z-score of $\leq-2.0$ ) to adjust for both the impact of nutritional status on susceptibility to infections [36] and potential unobserved confounding by socio-economic status. 
Missing covariate data were imputed using predictions from either multivariate normal regression, linear mixed effects models or Cox proportional hazards models, methods that are described in detail in the Supplementary Materials.

\subsection{Statistical Methods}

For all pathogen-specific associations, modified Poisson regression models were fitted to the binary outcome in the full database using generalized linear models with cluster-robust variance estimation to calculate adjusted risk ratios (RRs) for infection for each of the five risk factors in the presence of each other and the potential confounders [37]. This analysis did not attempt to attribute diarrheal episodes to particular etiological agents, and it was therefore possible (indeed common) for the same stool sample, including diarrheal samples, to be positive for multiple pathogens or negative for all pathogens. The pooled database was treated as longitudinal in structure and the GEMS and RECODISA subjects' statuses as cases or controls were only accounted for in the analysis insofar as they determined whether they contributed diarrheal or surveillance samples. To assess their associations with all-cause diarrhea, the household-level exposures were included with covariates in Cox proportional hazards models treating the subjects' age as survival time, reporting of a diarrheal episode as failure events and allowing for multiple failures per subject [9]. The Cox models were fitted only to the MAL-ED data since it was the only one of the five studies that collected data in a format suitable for survival analysis. Coefficients estimated from the models were visualized in dot-and-whisker plots. The crowding variable was coded with "crowded" households as the comparison group, so that a RR estimate of $<1$ could be interpreted in same way as the other 4 main exposures-as a protective effect of a factor which might conceivably be the target of an intervention. As a sensitivity analysis we repeated the analysis on the data from each contributing study in turn and compared these single-study results to the pooled findings. Analyses were carried out using Stata 16 [38].

\section{Results}

Table 1 gives the number and proportion of stool samples that were positive for the different species of enteropathogens in the 22 study sites and overall. Results were available for 50,000-57,000 stool samples depending on the pathogen, and overall positivity rates varied from $1.4 \%$-Salmonella spp. to $50.8 \%$-EAEC.

Table 2 shows the prevalence of the five household-level exposures in each of the study sites (before imputation of missing values and exclusion of samples not diagnosed with qPCR and including both GEMS1 and GEMS1A subjects-percent missing data by site is shown in supplementary Table S1). There was near universal coverage of improved drinking water sources among subjects in the sites in South Asia (with the exception of Karachi, Pakistan) and Brazil (with the exception of Fortaleza), while the site at Haydom, Tanzania, had by far the lowest coverage. The proportion of subjects living in households with access to improved sanitation facilities ranged from $0.0 \%$ in Haydom, to 97.0\% in Patos, Brazil. Haydom also had the lowest proportion of households with covered floors, followed by Nyanza, Kenya, and Mirzapur, Bangladesh, while in the urban sites of Brazil, India, and Mali, coverage of improved flooring was high. The highest levels of caregiver primary education completion were seen at the sites in Crato, Brazil; Midlands, Zimbabwe; and Venda, South Africa, while again prevalence for this variable was lowest in Haydom, Tanzania. The site with by far the highest prevalence of household crowding ( $\geq 3$ residents per bedroom) was Basse, The Gambia, while Nyanza, Kenya and Vellore, India had the lowest levels of crowding. It was not possible to calculate the crowding variable directly for the RECODISA and SHINE study sites. In both studies respondents were asked the number of residents per household, but in SHINE, no information was collected on the number of bedrooms, and in RECODISA, the number of rooms overall was recorded, but not the number of rooms for sleeping specifically. The process for imputing these and other missing data are explained in detail in the Supplementary Materials. There was very low correlation between the five exposure variables both within sites and in the pooled data ( $r=-0.25$ to 0.25$)$. The prevalence of the 
time-varying covariates among subjects at the time of enrollment (feeding status, and moderate-severe stunting and underweight) are shown in Table S3 in the Supplementary Materials.

Figure 2 plots the RRs for detection of specific enteric pathogen species and taxa in stool samples associated with the five household-level risk factors from Cox proportional hazard and generalized linear Poisson models adjusting for study site, age, sample type and feeding and nutritional status. Living in a household with an improved drinking water source was associated with a slightly statistically significant $17 \%$ reduction in the risk of diarrhea of any etiology (hazard ratio $(\mathrm{HR})=0.83$ $(0.71,0.97))$, but a similarly significant increase in the risk of astrovirus infection of $15 \%(R R=1.15$ $(1.00,1.33))$. Neither protozoa showed a significant association with having an improved water source; however, this variable did reduce the risk of infection with Aeromonas spp. by a moderately statistically significant $21 \%(R R=0.79(0.67,0.94))$ and EAEC by a slightly statistically significant $8 \%(R R=0.92$ $(0.85,0.99))$.

Household access to an improved sanitation facility was associated with a slightly statistically significant $9 \%$ reduction in risk of diarrhea $(\mathrm{HR}=0.91(0.83,0.99))$. Protective effects of improved sanitation were observed and were similar in magnitude for all included species of enteric pathogens with the one exception of Aeromonas spp. These estimates were statistically significant at the $\alpha \leq$ 0.05 level for two of the five virus species (astrovirus and norovirus), three of the bacterial infections (Campylobacter, EAEC, and typical EPEC) and for Cryptosporidium. Regarding the taxon-specific effects, improved sanitation afforded a moderately significant $12 \%$ reduction in risk of infection with any enteric virus $(R R=0.88(0.82,0.95))$ and a slightly significant $9 \%$ for either protozoa $(R R=0.91(0.83$, $1.00)$ ), and a highly statistically significant $19 \%$ reduction in the equivalent risk for any enteric bacteria $(\mathrm{RR}=0.81(0.72,0.90))$.

Living in a dwelling with a covered floor had the same association with the risk of diarrhea as sanitation-a moderately statistically significant $9 \%$ decrease $(\mathrm{HR}=0.91(0.85,0.98))$, but showed no significant associations with any enteric viral infections. Significant protective effects of improved flooring against numerous enteric bacteria species were observed ranging from a slightly statistically significant reduction in EAEC risk of $8 \%(R R=0.93(0.87,1.00))$ to a highly statistically significant $13 \%$ decreased risk of infection with Campylobacter spp. $(R R=0.87(0.81,0.94))$. One of the largest effects of this or any other variable was seen for infections with Giardia spp., a moderately statistically significant $16 \%$ reduction $(R R=0.84(0.76,0.94))$.

Having a primary caregiver who had completed primary education was associated with a slightly statistically significant increase in the risk of all-cause diarrhea of $7 \%(R R=1.07(1.00,1.14))$. Most other pathogen- and taxon-specific models showed a small protective effect of this exposure, which was slightly statistically significant for astrovirus $(R R=0.91(0.85,0.99))$, any enteric virus $(R R=0.94(0.89$, $0.98))$, typical EPEC $(\operatorname{RR}=0.92(0.86,0.98))$, Shigella spp./EIEC $(R R=0.92(0.85,0.98))$, and any enteric bacteria $(R R=0.92(0.85,0.98))$, moderately significant for Giardia spp. $(R R=0.87(0.79,0.95))$, and any enteric protozoa $(R R=0.91(0.86,0.978))$, and highly so for Campylobacter spp. $(R R=0.88(0.82,0.94))$.

Living in a household with fewer than three residents per bedroom was associated with a slightly statistically significant $7 \%$ lower risk of all-cause diarrhea $(R R=0.93(0.88,0.99)), 5 \%$ for any enteric virus $(R R=0.95(0.88,0.99))$, and $7 \%$ for any Campylobacter spp. $(R R=0.93(0.88,0.99))$. 
Table 1. Number and percentages (\%) of stool samples from children aged 0-59 months that were positive for different species of enteropathogens by qPCR in 22 study sites and overall (includes both cases and controls from the Global Enteric Multicenter Study (GEMS)1 and RECODISA).

\begin{tabular}{|c|c|c|c|c|c|c|c|c|}
\hline & $\begin{array}{c}\text { Adenovirus } \\
40 / 41\end{array}$ & Astrovirus & Norovirus & Rotavirus & Sapovirus & Aeromonas spp. & Campylo-bacter spp. & EAEC \\
\hline Bamako, Mali & $460(26.3)$ & $136(7.7)$ & $351(19.9)$ & $216(12.3)$ & $238(13.5)$ & $41(2.3)$ & $899(50.8)$ & $1151(65.1)$ \\
\hline Basse, The Gambia & $373(25.4)$ & $98(6.4)$ & $250(16.3)$ & $227(14.8)$ & $201(13.1)$ & $19(1.3)$ & $787(52.2)$ & $873(58.7)$ \\
\hline Bhaktapur, Nepal & $348(5.9)$ & $301(5.1)$ & $714(12.0)$ & $249(4.2)$ & 651 (11.1) & $149(2.5)$ & $1256(22.3)$ & $2690(47.0)$ \\
\hline Cajazeiras, Brazil & $1(0.5)$ & $9(4.5)$ & $8(4.0)$ & $12(6.0)$ & $9(4.5)$ & $6(3.0)$ & $6(3.0)$ & $68(34.0)$ \\
\hline Crato, Brazil & $5(2.5)$ & $4(2.0)$ & $2(1.0)$ & $46(23.0)$ & $6(3.0)$ & $12(6.0)$ & $26(13.0)$ & $83(41.5)$ \\
\hline Dhaka, Bangladesh & $1306(23.5)$ & $1142(20.3)$ & $1086(19.0)$ & $578(10.2)$ & $1054(18.8)$ & $162(2.8)$ & $1730(33.8)$ & $2245(41.1)$ \\
\hline Fortaleza, Brazil & $137(4.7)$ & $52(1.8)$ & $187(6.4)$ & $40(1.5)$ & $132(4.5)$ & $40(1.4)$ & $401(13.8)$ & $883(30.1)$ \\
\hline Haydom, Tanzania & $365(8.3)$ & $282(6.4)$ & 735 (16.6) & $227(5.2)$ & $482(11.0)$ & $131(3.0)$ & $1818(45.9)$ & $2843(65.1)$ \\
\hline Karachi, Pakistan & $366(22.4)$ & 191 (11.6) & $393(23.8)$ & $161(9.8)$ & 306 (18.5) & $122(7.5)$ & $1023(62.9)$ & $1132(68.9)$ \\
\hline Kolkota, India & 734 (41.5) & $101(5.8)$ & $316(18.0)$ & $321(18.3)$ & 201 (11.4) & $193(10.9)$ & $824(46.6)$ & $1028(58.1)$ \\
\hline Loreto, Peru & $1380(21.0)$ & 1049 (15.7) & $1230(18.0)$ & $261(4.0)$ & $975(14.9)$ & $177(2.6)$ & $1293(20.3)$ & $3174(55.6)$ \\
\hline Manhiça, Mozambique & 377 (36.3) & $41(4.0)$ & $151(14.7)$ & $202(19.7)$ & $120(11.7)$ & $50(4.8)$ & $462(44.5)$ & 817 (78.7) \\
\hline Midlands, Zimbabwe & $198(8.3)$ & $66(2.8)$ & 277 (11.7) & $122(5.1)$ & $155(6.5)$ & $36(1.5)$ & $602(25.5)$ & $1361(57.6)$ \\
\hline Mirzapur, Bangladesh & $443(24.7)$ & $89(5.0)$ & $243(13.6)$ & $392(21.9)$ & 183 (10.2) & $59(3.3)$ & 434 (24.2) & 992 (55.3) \\
\hline N. Feroze, Pakistan & $823(12.8)$ & 930 (14.6) & 1388 (21.4) & $274(4.2)$ & $1063(16.6)$ & $35(0.5)$ & $1667(27.0)$ & 2288 (36.5) \\
\hline Nyanza, Kenya & $165(9.1)$ & $61(3.4)$ & $250(13.9)$ & $160(9.0)$ & $179(10.0)$ & $109(6.0)$ & $662(36.5)$ & 952 (53.1) \\
\hline Ouricuri, Brazil & $7(3.5)$ & $9(4.5)$ & $12(6.0)$ & $5(2.5)$ & $6(3.0)$ & $11(5.5)$ & $17(8.5)$ & $52(26.1)$ \\
\hline Patos, Brazil & $6(3.0)$ & $0(0.0)$ & $4(2.0)$ & $6(3.0)$ & $1(0.5)$ & $3(1.5)$ & $10(5.0)$ & $194(97.0)$ \\
\hline Picos, Brazil & $3(1.8)$ & $0(0.0)$ & $0(0.0)$ & $14(8.5)$ & $8(4.9)$ & $2(1.0)$ & $15(7.5)$ & $92(46.0)$ \\
\hline Souza, Brazil & $2(1.0)$ & $0(0.0)$ & $10(5.1)$ & $3(1.5)$ & $5(2.5)$ & $2(1.1)$ & $12(6.3)$ & $161(83.9)$ \\
\hline Vellore, India & 921 (17.1) & $622(11.6)$ & $822(15.2)$ & $434(8.1)$ & $789(14.7)$ & $275(5.1)$ & $1144(22.0)$ & $3283(62.5)$ \\
\hline Venda, South Africa & $503(10.7)$ & $332(7.1)$ & $529(11.2)$ & $92(2.1)$ & $517(11.0)$ & $24(0.5)$ & 535 (11.4) & 1698 (36.0) \\
\hline Total positive & 8923 (15.7) & 5515 (9.7) & 8958 (15.6) & $4042(7.2)$ & $7281(12.8)$ & $1658(2.9)$ & $15,623(28.4)$ & $28,060(50.8)$ \\
\hline Total stools & 56,704 & 56,828 & 57,350 & 56,168 & 56,668 & 57,185 & 54,923 & 55,280 \\
\hline
\end{tabular}


Table 1. Cont.

\begin{tabular}{|c|c|c|c|c|c|c|c|c|}
\hline & $\begin{array}{c}\text { Adenovirus } \\
40 / 41\end{array}$ & Astrovirus & Norovirus & Rotavirus & Sapovirus & Aeromonas spp. & Campylo-bacter spp. & EAEC \\
\hline & Atypical EPEC & Typical EPEC & LT-ETEC & ST-ETEC & Salmonella spp. & $\begin{array}{c}\text { Shigella } \\
\text { spp./EIEC }\end{array}$ & Crypto-sporidium spp. & Giardia spp \\
\hline Bamako, Mali & 382 (21.6) & 588 (33.2) & $511(29.0)$ & 319 (18.1) & $52(2.9)$ & $567(32.1)$ & $522(29.5)$ & $1233(70.8)$ \\
\hline Basse, The Gambia & $330(22.0)$ & $461(30.8)$ & $370(24.8)$ & $289(19.3)$ & $82(5.5)$ & $480(32.7)$ & 279 (18.5) & $630(42.4)$ \\
\hline Bhaktapur, Nepal & $1673(28.5)$ & $403(6.8)$ & $575(9.7)$ & $620(10.5)$ & $55(0.9)$ & $376(6.3)$ & $272(4.6)$ & $545(10.3)$ \\
\hline Cajazeiras, Brazil & $34(17.0)$ & $3(1.5)$ & $7(3.5)$ & $0(0.0)$ & $7(3.5)$ & $10(5.1)$ & $24(12.1)$ & $66(33.3)$ \\
\hline Crato, Brazil & $66(33.0)$ & $13(6.5)$ & $33(16.5)$ & $0(0.0)$ & $78(39.0)$ & $76(38.0)$ & $13(6.5)$ & $55(27.5)$ \\
\hline Dhaka, Bangladesh & $1328(23.6)$ & 1075 (19.1) & $849(15.0)$ & $1799(32.6)$ & $57(1.0)$ & 865 (15.4) & $380(6.8)$ & $661(12.8)$ \\
\hline Fortaleza, Brazil & $764(25.9)$ & $100(3.4)$ & $129(4.4)$ & $73(2.5)$ & $27(0.9)$ & $158(5.4)$ & $37(1.3)$ & $266(9.7)$ \\
\hline Haydom, Tanzania & $1266(28.6)$ & 821 (18.6) & $1141(26.1)$ & $1242(28.3)$ & $19(0.4)$ & $790(17.9)$ & $514(12.0)$ & 931 (27.3) \\
\hline Karachi, Pakistan & $358(21.8)$ & $545(33.2)$ & $404(24.7)$ & $356(21.8)$ & $22(1.3)$ & $543(32.9)$ & $383(23.4)$ & $931(57.0)$ \\
\hline Kolkota, India & 519 (29.4) & $367(20.8)$ & $392(22.2)$ & $293(16.6)$ & $23(1.3)$ & $533(30.1)$ & 275 (15.6) & $1076(62.2)$ \\
\hline Loreto, Peru & $1548(24.1)$ & $807(12.1)$ & 1137 (17.2) & $763(11.4)$ & $85(1.3)$ & $786(11.8)$ & $633(9.6)$ & $1415(26.1)$ \\
\hline Manhiça, Mozambique & $279(26.9)$ & $318(30.7)$ & $280(27.0)$ & $329(31.7)$ & $41(4.0)$ & 328 (31.6) & $323(31.2)$ & 726 (70.9) \\
\hline Midlands, Zimbabwe & $608(25.7)$ & $219(9.2)$ & 464 (19.6) & $231(9.7)$ & $42(1.8)$ & $93(3.9)$ & $210(8.9)$ & 348 (15.1) \\
\hline Mirzapur, Bangladesh & $430(24.0)$ & 209 (11.6) & 337 (18.8) & $95(5.3)$ & $22(1.2)$ & $648(36.1)$ & $80(4.5)$ & 377 (21.1) \\
\hline N. Feroze, Pakistan & 869 (13.4) & $683(10.6)$ & $578(8.9)$ & 605 (9.4) & $4(0.1)$ & $445(6.9)$ & $402(6.3)$ & 1585 (34.4) \\
\hline Nyanza, Kenya & $453(25.0)$ & $440(24.3)$ & $555(31.0)$ & 278 (15.3) & $39(2.1)$ & $421(23.2)$ & 336 (18.5) & $735(40.8)$ \\
\hline Ouricuri, Brazil & 27 (13.6) & $1(0.5)$ & $6(3.0)$ & $0(0.0)$ & $28(14.0)$ & $22(11.0)$ & $1(0.5)$ & $22(11.0)$ \\
\hline Patos, Brazil & $76(38.0)$ & $4(2.0)$ & $17(8.5)$ & $7(3.5)$ & $13(6.5)$ & $8(4.0)$ & $10(5.0)$ & $21(10.6)$ \\
\hline Picos, Brazil & $21(10.5)$ & $5(2.5)$ & $5(2.5)$ & $0(0.0)$ & $26(13.0)$ & $21(10.5)$ & $11(5.5)$ & $58(29.0)$ \\
\hline Souza, Brazil & $86(44.8)$ & $3(1.6)$ & $12(6.3)$ & $5(2.6)$ & $17(8.9)$ & $10(5.3)$ & $17(8.9)$ & $30(15.8)$ \\
\hline Vellore, India & $1440(26.7)$ & $870(16.2)$ & 877 (16.4) & $717(13.4)$ & $67(1.2)$ & 701 (13.0) & $278(5.2)$ & $1039(23.2)$ \\
\hline Venda, South Africa & $945(20.1)$ & $212(4.5)$ & $346(7.4)$ & $194(4.2)$ & $14(0.3)$ & $337(7.2)$ & $226(4.9)$ & 704 (16.2) \\
\hline Total positive & $13,502(23.8)$ & 8147 (14.3) & 9025 (15.9) & 8215 (14.5) & $820(1.4)$ & $8218(14.4)$ & $5226(9.3)$ & $13,454(26.8)$ \\
\hline Total stools & 56,713 & 56,943 & 56,832 & 56,842 & 57,154 & 56,930 & 56,485 & 50,177 \\
\hline
\end{tabular}


Table 2. Prevalence of 5 household-level exposures among study subjects aged 0-59 months in 22 study sites (before excluding samples not diagnosed with qPCR).

\begin{tabular}{|c|c|c|c|c|c|c|}
\hline & $\begin{array}{l}\text { Improved Water } \\
\text { Source }\end{array}$ & Improved Sanitation & Improved Flooring & Caregiver Education & $\begin{array}{l}\text { Household } \\
\text { Crowding }\end{array}$ & Total Subjects \\
\hline Bamako, Mali & $5872(87.5)$ & $127(1.9)$ & $5253(98.5)$ & $1242(31.4)$ & $3119(58.5)$ & 6711 \\
\hline Basse, The Gambia & 4087 (86.3) & $82(1.7)$ & $2837(85.0)$ & $1029(42.4)$ & $2987(89.5)$ & 4738 \\
\hline Bhaktapur, Nepal & $232(98.3)$ & $131(55.5)$ & $104(44.1)$ & $152(64.4)$ & $47(19.9)$ & 240 \\
\hline Cajazeiras, Brazil & $172(86.0)$ & $197(98.5)$ & $198(99.0)$ & $137(68.5)$ & - & 200 \\
\hline Crato, Brazil & $189(94.5)$ & $181(90.5)$ & $170(87.2)$ & $169(84.9)$ & - & 200 \\
\hline Dhaka, Bangladesh & $242(100.0)$ & $28(11.6)$ & $226(93.4)$ & $57(23.6)$ & $3(1.2)$ & 265 \\
\hline Fortaleza, Brazil & $142(67.6)$ & $201(95.7)$ & $208(99.0)$ & $146(69.5)$ & $6(2.9)$ & 233 \\
\hline Haydom, Tanzania & $79(31.6)$ & $0(0.0)$ & $17(6.8)$ & $6(2.4)$ & $23(9.2)$ & 262 \\
\hline Karachi, Pakistan & $3274(62.6)$ & $2428(46.4)$ & $2881(75.6)$ & $1120(37.8)$ & $443(11.6)$ & 5231 \\
\hline Kolkata, India & 5147 (98.7) & $635(12.2)$ & 3877 (95.9) & $1880(64.2)$ & $160(4.0)$ & 5214 \\
\hline Loreto, Peru & $309(89.6)$ & $70(20.3)$ & $98(28.4)$ & $192(56.3)$ & $45(13.0)$ & 378 \\
\hline $\begin{array}{l}\text { Manhiça, } \\
\text { Mozambique }\end{array}$ & $2745(85.1)$ & $219(6.8)$ & $1691(70.1)$ & $454(24.7)$ & $417(17.4)$ & 3227 \\
\hline Midlands, Zimbabwe & $611(61.8)$ & $619(61.2)$ & $547(55.2)$ & $844(82.6)$ & - & 1046 \\
\hline Mirzapur, Bangladesh & $5907(99.8)$ & $2830(47.8)$ & $887(20.8)$ & $2662(75.3)$ & $769(18.0)$ & 5916 \\
\hline $\begin{array}{l}\text { Naushahro Feroze, } \\
\text { Pakistan }\end{array}$ & 265 (100.0) & $9(3.4)$ & $74(27.9)$ & $41(15.5)$ & $55(20.8)$ & 277 \\
\hline Nyanza, Kenya & $2549(64.5)$ & $169(4.3)$ & $659(19.5)$ & $1630(52.9)$ & $34(1.0)$ & 3951 \\
\hline Ouricuri, Brazil & $193(96.5)$ & 195 (97.5) & $195(98.0)$ & $142(71.0)$ & - & 200 \\
\hline Patos, Brazil & $199(100.0)$ & $197(98.5)$ & $198(99.5)$ & $139(70.6)$ & - & 200 \\
\hline Picos, Brazil & $198(99.5)$ & $193(96.5)$ & $192(96.5)$ & $129(64.5)$ & - & 200 \\
\hline Souza, Brazil & $200(100.0)$ & $181(90.5)$ & $198(99.0)$ & $125(62.5)$ & - & 200 \\
\hline Vellore, India & $235(100.0)$ & $12(5.1)$ & $220(93.6)$ & $123(52.3)$ & $1(0.4)$ & 251 \\
\hline Venda, South Africa & $216(85.4)$ & $3(1.2)$ & $233(92.1)$ & $207(81.8)$ & $62(24.5)$ & 314 \\
\hline
\end{tabular}




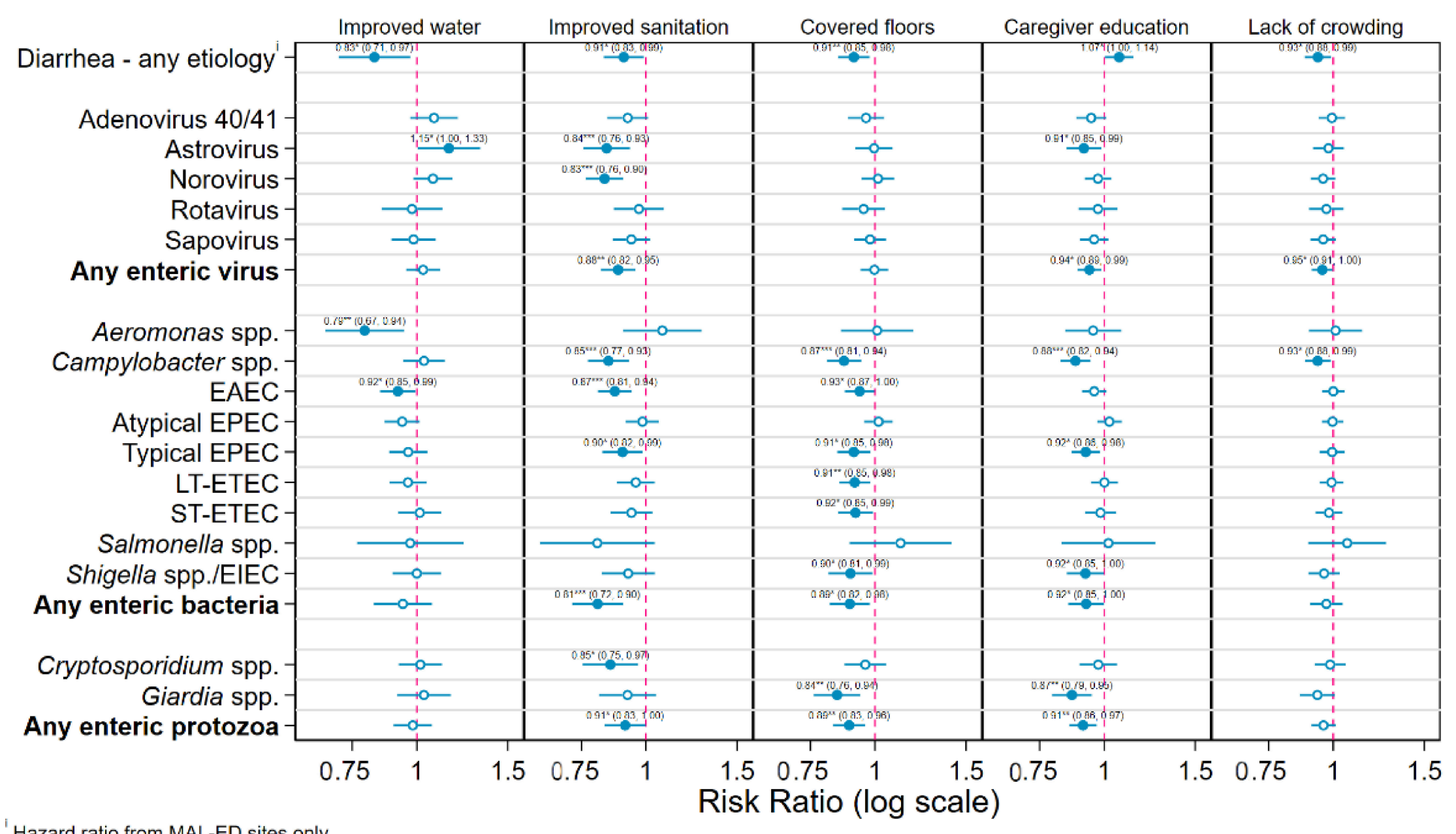

Hazard ratio from MAL-ED sites only

${ }_{* *} p<0.001,{ }^{* *} p=0.001-0.01,{ }^{*} p=0.01-0.05$

Figure 2. Hazard and risk ratios for detection of specific enteric pathogen species in stool samples associated with 5 household-level risk factors from Cox and generalized linear models adjusting for study site, age, sample type and feeding and nutritional status. 


\section{Discussion}

During early childhood a large proportion of time is spent within the family dwelling, and therefore characteristics of the household and caregivers contribute substantially to a child's health status, especially with regard to diarrheal disease and its sequelae. Features of the household environment may serve to promote or interrupt the transmission of diarrhea-causing pathogens via the fecal-oral and other routes. Researching these effects can be challenging, however, because studies of diarrheal disease outcomes, particularly those that make comparisons across multiple populations, may be biased by differences in caregiver reporting and understanding of diarrhea symptomatology. Detections of specific enteric pathogens from stool samples can serve as more objective, generalizable morbidity metrics for evaluating the impact of specific factors on risk of contracting disease and are increasingly being reported by trials and observational studies [39]. This was illustrated in recently published results from the SHINE trial, which showed a statistically significant decrease in parasite detections following water, sanitation, and hygiene (WASH) interventions [29] in the absence of any commensurate detectable decrease in caregiver-reported diarrhea [40]. Nonetheless, several location-specific attempts to quantify the separate effects of household characteristics on the transmission of particular enteric pathogens have yielded effect sizes that are small, non-significant, or with inconsistent directions [22,29,41].

To address these limitations this analysis combined results from several, rigorous population-based studies that each used highly sensitive, broad spectrum molecular diagnostics, and standardized exposure definitions. The resulting dataset-some 58,000 stool samples from 15,000 subjects in 22 locations - is of a size and diversity of epidemiologic contexts that is unparalleled in the study of enteric pathogens. While many of the findings appear to confirm prevailing assumptions about the transmission pathways of the analyzed pathogens, others are suggestive of hitherto novel hypotheses.

Household use of an improved water source had the largest protective effect against all-cause diarrhea of any of the five exposures. However, the direction of the association of this exposure with particular pathogens was inconsistent and, in almost all cases, non-significant. It has been suggested that sanitation interventions may be more likely to interrupt transmission of bacteria and parasites than viruses which have more environmentally mediated transmission routes [22]. However, there are known exceptions to this, and in fact, this analysis found protective effects of improved sanitation that were remarkably consistent across all pathogen species and taxa with most statistically significant effects falling within a range of around a 10-20\% reduction in detection risk. While Aeromonas spp. infection risk was particularly reduced by improved water source, a biologically plausible finding for a pathogen that lives freely in sea and surface waters, the clinical importance of that finding is unclear. Although evidence has been published of Aeromonas-associated morbidity in specific contexts [42] (e.g., some South Asian GEMS sites [2]), it is not broadly believed to be an enteropathogen with a high attributable disease burden [7].

Having covered floors in the dwelling had the same effect on all-cause diarrhea as improved sanitation and to a higher level of statistical significance. Pathogen-specific estimates however show a distinct pattern of viruses being unaffected by this exposure, while consistent protective effects against bacterial and protozoal infections are evident. It is notable that the decrease in infection risk was most marked for the zoonotic enteropathogens Campylobacter and Giardia, given that many of the study sites contributing data to this analysis are in settings characterized by high levels of household-scale livestock husbandry, as well as children sharing the peridomestic space and even their sleeping quarters (the intradomiciliary space) with animals [43]. We hypothesize that investment in improved flooring materials that are easily disinfected, such as cement or tiles, can both impede the survival or transmissibility of pathogens [44] while also incentivizing the transfer of animals to predominantly outside the domestic space. For instance, the considerable decrease in infection risk for Campylobacter spp. can be linked to a hypothesized decrease in exposure to avian feces once improved flooring is introduced [45], as well as a decreased survival of Campylobacter in non-soil surfaces that are easily cleaned and dried [46-48]. It should be noted that some of the pathogens demonstrating risk 
reductions are not zoonotic in origin (e.g., Shigella and E. coli pathotypes) suggesting that improved flooring diminished transmission from human as well as animal sources.

Lack of crowding also showed a protective effect against the two zoonotic pathogens Campylobacter and Giardia. This too may be a marker of proximity to livestock, with children living in crowded households more likely to be forced to share their living or sleeping quarters with animals [43,49-51]. It is a limitation of this analysis that it did not include household livestock ownership among the potential exposures. This was due to heterogeneity in the ways in which this information was recorded across the different studies. The apparent increase in risk conferred by caregiver education on diarrhea of any etiology runs counter to a priori assumptions and may be an artifact of differential caregiver reporting of their child's symptoms by education status in the MAL-ED cohorts (i.e., that mothers with higher educational attainment are more likely to report their child's diarrheal episode to a study fieldworker when it occurs).

Another limitation inherent to the analysis of observational data, is that the role of residual confounding, in this case by socio-economic status, which can be expected to be associated with both the exposures and the outcomes, cannot be ruled out. By adjusting each of the household exposures for each other and for the two anthropometric indicators-childhood nutritional status being highly correlated with household socio-economic indicators [52] — we hoped to control for such confounding as much as possible; however, in an analysis of a dataset of this size capable of detecting small effect sizes, results should be interpreted with caution and in light of the possibility of residual confounding. Other more precise, composite indicators of household socio-economic status derived from income and asset ownership are available [52], but the necessary information was not collected uniformly across contributing studies. Future studies into this topic might consider employing causal inference methods such as propensity score weighting or matching to further adjust for confounding [53]. Another potential source of residual confounding in analyses of pooled data is differences in the ascertainment of the exposures and outcomes across the included studies due to variation in their design and the instruments used. By restricting the data to samples analyzed by highly sensitive molecular diagnostics and exposures that are widely used and reported as indicators of socio-economic development, we have attempted to minimize the possibility that such differences might lead to spurious inferences. Indeed, a sensitivity analysis (not reported) revealed single-study findings that were broadly consistent with the pooled results, taking account of the loss of statistical power from restricting the number of observations.

While systematic reviews of household-level interventions report pooled protective effects of $25-75 \%$ for diarrheal outcomes [13,54], recent single-site trials have found at best only qualified impacts [15]. The small protective effects identified in this study-reductions in risk of around $6-20 \%$ for any exposure/outcome combination - fall towards the latter, more modest end of the range of estimates. These pathogen-specific associations also appear modest in comparison to intra-subject-level exposures, such as rotavirus vaccine status- $50 \%$ protective against severe rotavirus morbidity [55]—and maternal FUT2 secretor status—a 37\% reduction in LT-ETEC infection [9]. Data inputs for systematic reviews skew preferentially toward efficacy-type study designs, measuring effects of delivering an intervention under trial conditions which may not be reflective of true community transmission dynamics. Apart from the $4 \%$ contributed by the SHINE trial, the data used in this analysis were from observational studies that did not seek to test an intervention. There is increasing evidence that under such real-world conditions, traditional, low-cost improvements to housing conditions (such as the installation of ventilated pit latrines) may be inadequate, and that the community is better thought of as the true unit of exposure [56]. Under this hypothesis, only an ambitious, transformative WASH agenda involving wide-ranging investments in community-level water, wastewater, housing, and livestock infrastructure and management can meet the needs for improving child health and eliminating pathogen exposure $[15,56]$. 


\section{Conclusions}

In conclusion, a diverse array of enteropathogens are endemic and highly prevalent across distinct geographical and epidemiological settings, notably Campylobacter, EAEC, Giardia, and Atypical EPEC, as has been reported elsewhere. Improvements in sanitation may reduce diarrheal disease by suppressing transmission of enteropathogens of all three taxa, while improvements in flooring may do so specifically through decreases in enteric bacteria and protozoa infection without interrupting virus transmission, with the reverse being the case for reducing household crowding. While drinking water source improvements also appear to reduce diarrhea risk, it is not clear from these findings which pathogen taxa mediate this association. By combining data collected at multiple epidemiologically and socioeconomically diverse sites, this analysis was able to quantify the associations between each of five household-level exposures on the risk of specific enteric infections, effects which differed by pathogen species and taxa but were broadly consistent with hypothesized transmission mechanisms. With further research, such estimates may be used within expanded WASH programs to target interventions to the particular pathogen profiles of individual communities and prioritize resources accordingly.

Supplementary Materials: The following are available online at http://www.mdpi.com/1660-4601/17/21/8078/s1, Missing data; Table S1: Percent missing data for household-level variables used in imputation by study, Table S2: Mapping of exposure variable definitions used by included studies onto published definitions, Table S3: Prevalence of time-varying covariates among study subjects at enrollment in 22 study sites.

Author Contributions: Conceptualization, J.M.C., B.F.Z. and M.N.K.; methodology, J.M.C.; formal analysis, J.M.C.; investigation, A.S.G.F., M.J.H., D.S., S.K., I.M., A.K.M.Z., M.I.N., R.O., R.F.B., S.O.S., M.M.L., K.L.K., T.A., P.B., Z.B., E.M., P.P.Y., P.S.S., M.P.O., G.K., A.A.M.L., J.H. and A.P.; data curation, J.M.C. and A.R.; writing-original draft preparation, J.M.C.; writing-review and editing, M.N.K. and F.S.; visualization, J.M.C.; supervision, M.N.K.; project administration, J.M.C.; funding acquisition, B.F.Z. and M.N.K. All authors have read and agreed to the published version of the manuscript.

Funding: The Etiology: Risk Factors, and Interactions of Enteric Infections and Malnutrition and the Consequences for Child Health and Development Project (MAL-ED) is carried out as a collaborative project supported by the Bill \& Melinda Gates Foundation (47075), the Foundation for the National Institutes of Health, and the National Institutes of Health, Fogarty International Center. Additional support for MAL-ED was obtained from the BMGF (Improved Biomarkers of Environmental Enteropathy, OPP1066146) and the Sherrilyn and Ken Fisher Center for Environmental Infectious Diseases of the Johns Hopkins School of Medicine (to MNK). The Global Enteric Multicenter Study (GEMS) was supported by grants 38774 and OPP1033572 from the Bill \& Melinda Gates Foundation. The SHINE trial is funded by the Bill \& Melinda Gates Foundation (OPP1021542 to Johns Hopkins Bloomberg School of Public Health and OPP1143707 to Zvitambo Institute for Maternal and Child Health Research), the UK Department for International Development, the Wellcome Trust (093768/Z/10/Z and 108065/Z/15/Z), the Swiss Agency for Development and Cooperation (8106727), UNICEF (PCA-2017-0002), and the US National Institutes of Health (R01 HD060338/HD/NICHD). The research presented in this article was supported financially by NASA's Group on Earth Observations Work Programme (16-GEO16-0047) and The National Institutes of Health's National Institute of Allergy and Infectious Diseases grant 1R03AI151564-01.

Acknowledgments: We thank the participants, their families, and the study communities for their dedicated time and effort to better the understanding the transmission and more enduring impact of enteric infections in early childhood on the health of individuals and populations. Ciara E. O'Reilly and Eric D. Mintz of the US Centers for Disease Control and Prevention provided comments on the manuscript.

Conflicts of Interest: The authors declare no conflict of interest. The funders had no role in the design of the study; in the collection, analyses, or interpretation of data; in the writing of the manuscript, or in the decision to publish the results.

\section{References}

1. Liu, L.; Oza, S.; Hogan, D.; Chu, Y.; Perin, J.; Zhu, J.; Lawn, J.E.; Cousens, S.; Mathers, C.; Black, R.E. Global, regional, and national causes of under-5 mortality in 2000-15: An updated systematic analysis with implications for the Sustainable Development Goals. Lancet 2016, 388, 3027-3035. [CrossRef]

2. Kotloff, K.; Nataro, J.P.; Blackwelder, W.C.; Nasrin, D.; Farag, T.H.; Panchalingam, S.; Wu, Y.; Sow, S.; Sur, D.; Breiman, R.F.; et al. Burden and aetiology of diarrhoeal disease in infants and young children in developing countries (the Global Enteric Multicenter Study, GEMS): A prospective, case-control study. Lancet 2013, 382, 209-222. [CrossRef] 
3. Troeger, C.; Forouzanfar, M.; Rao, P.C.; Khalil, I.; Brown, A.; Reiner, R.C.; Fullman, N.; Thompson, R.L.; Abajobir, A.; Ahmed, M.B.; et al. Estimates of global, regional, and national morbidity, mortality, and aetiologies of diarrhoeal diseases: A systematic analysis for the Global Burden of Disease Study 2015. Lancet Infect. Dis. 2017, 17, 909-948. [CrossRef]

4. McGovern, M.E.; Krishna, A.; Aguayo, V.M.; Subramanian, S.V. A review of the evidence linking child stunting to economic outcomes. Int. J. Epidemiol. 2017, 46, 1171-1191. [CrossRef] [PubMed]

5. Dewey, K.G.; Begum, K. Long-term consequences of stunting in early life. Matern. Child Nutr. 2011, 7, 5-18. [CrossRef]

6. Kotloff, K.L. The Burden and Etiology of Diarrheal Illness in Developing Countries. Pediatr. Clin. North Am. 2017, 64, 799-814. [CrossRef] [PubMed]

7. Platts-Mills, J.A.; Liu, J.; Rogawski, E.T.; Kabir, F.; Lertsethtakarn, P.; Siguas, M.; Khan, S.S.; Praharaj, I.; Murei, A.; Nshama, R.; et al. Use of quantitative molecular diagnostic methods to assess the aetiology, burden, and clinical characteristics of diarrhoea in children in low-resource settings: A reanalysis of the MAL-ED cohort study. Lancet Glob. Health 2018, 6, e1309-e1318. [CrossRef]

8. Lima, A.A.; Oliveira, D.B.; Quetz, J.S.; Havt, A.; Prata, M.M.G.; Lima, I.F.N.; Soares, A.M.; Filho, J.Q.; Lima, N.L.; Medeiros, P.H.Q.S.; et al. Etiology and severity of diarrheal diseases in infants at the semiarid region of Brazil: A case-control study. PLoS Negl. Trop. Dis. 2019, 13, e0007154. [CrossRef]

9. Colston, J.M.; Francois, R.; Pisanic, N.; Yori, P.P.; McCormick, B.J.J.; Olortegui, M.P.; Gazi, A.; Svensen, E.; Ahmed, M.M.M.; Mduma, E.; et al. Effects of Child and Maternal Histo-Blood Group Antigen Status on Symptomatic and Asymptomatic Enteric Infections in Early Childhood. J. Infect. Dis. 2019, 220, 151-162. [CrossRef] [PubMed]

10. Chao, D.L.; Roose, A.; Roh, M.K.; Kotloff, K.L.; Proctor, J.L. The seasonality of diarrheal pathogens: A retrospective study of seven sites over three years. PLoS Negl. Trop. Dis. 2019, 13, e0007211. [CrossRef] [PubMed]

11. Eisenberg, J.N.S.; Trostle, J.; Sorensen, R.J.; Shields, K.F. Toward a systems approach to enteric pathogen transmission: From individual independence to community interdependence. Annu. Rev. Public Health 2012, 33, 239-257. [CrossRef] [PubMed]

12. Lopman, B.; Gastañaduy, P.; Park, G.W.; Hall, A.J.; Parashar, U.D.; Vinjé, J. Environmental transmission of norovirus gastroenteritis. Curr. Opin. Virol. 2012, 2, 96-102. [CrossRef]

13. Wolf, J.; Hunter, P.R.; Freeman, M.C.; Cumming, O.; Clasen, T.; Bartram, J.; Higgins, J.P.T.; Johnston, R.; Medlicott, K.; Boisson, S.; et al. Impact of drinking water, sanitation and handwashing with soap on childhood diarrhoeal disease: Updated meta-analysis and meta-regression. Trop. Med. Int. Health 2018, 23, 508-525. [CrossRef] [PubMed]

14. Wolf, J.; Prüss-Ustün, A.; Cumming, O.; Bartram, J.; Bonjour, S.; Cairncross, S.; Clasen, T.; Colford, J.M., Jr.; Curtis, V.; De France, J.; et al. Systematic review: Assessing the impact of drinking water and sanitation on diarrhoeal disease in low- and middle-income settings: Systematic review and meta-regression. Trop. Med. Int. Health 2014, 19, 928-942. [CrossRef] [PubMed]

15. Cumming, O.; Arnold, B.F.; Ban, R.; Clasen, T.F.; Mills, J.E.; Freeman, M.C.; Gordon, B.; Guiteras, R.; Howard, G.; Hunter, P.R.; et al. The implications of three major new trials for the effect of water, sanitation and hygiene on childhood diarrhea and stunting: A consensus statement. BMC Med. 2019, 17, 173. [CrossRef]

16. Crawford, S.E.; Ramani, S.; Tate, J.E.; Parashar, U.D.; Svensson, L.; Hagbom, M.; Franco, M.A.; Greenberg, H.B.; O'Ryan, M.; Kang, G.; et al. Rotavirus infection. Nat. Rev. Dis. Prim. 2017, 3, 17084. [CrossRef] [PubMed]

17. Lanata, C.F.; Fischer-Walker, C.L.; Olascoaga, A.C.; Torres, C.X.; Aryee, M.J.; Black, R.E.; Child Health Epidemiology Reference Group of the World Health Organization and UNICEF. Global Causes of Diarrheal Disease Mortality in Children <5 years of age: A systematic review. PLoS ONE 2013, 8, e72788. [CrossRef]

18. Platts-Mills, J.A.; Rogawski, E.T.; Kosek, M.N.; Houpt, E.R. Aetiology, burden and clinical characteristics of diarrhoea in children in low-resource settings using quantitative molecular diagnostics: Results from the MAL-ED cohort study. Lancet Glob. Health 2018, in press. [CrossRef]

19. Braun-Falco, M.; Mankin, H.J.; Wenger, S.L.; Kendall, S.D.; Blobe, G.C.; Weber, C.K.; Lorenz, R.; Adler, G.; Whitcomb, D.C.; Yadav, D.; et al. PAP. In Encyclopedia of Molecular Mechanisms of Disease; Springer: Berlin/Heidelberg, Germany, 2009; p. 1573. 
20. Null, C.; Stewart, C.P.; Pickering, A.J.; Dentz, H.N.; Arnold, B.F.; Arnold, C.D.; Benjamin-Chung, J.; Clasen, T.; Dewey, K.G.; Fernald, L.C.H.; et al. Effects of water quality, sanitation, handwashing, and nutritional interventions on diarrhoea and child growth in rural Kenya: A cluster-randomised controlled trial. Lancet Glob. Health 2018, 6, e316-e329. [CrossRef]

21. Exum, N.G.; Olórtegui, M.P.; Yori, P.P.; Davis, M.F.; Heaney, C.D.; Kosek, M.N.; Schwab, K.J. Floors and Toilets: Association of Floors and Sanitation Practices with Fecal Contamination in Peruvian Amazon Peri-Urban Households. Environ. Sci. Technol. 2016, 50, 7373-7381. [CrossRef]

22. Knee, J.; Sumner, T.; Adriano, Z.; Berendes, D.; De Bruijn, E.; Schmidt, W.-P.; Nalá, R.; Cumming, O.; Brown, J. Risk factors for childhood enteric infection in urban Maputo, Mozambique: A cross-sectional study. PLoS Negl. Trop. Dis. 2018, 12, e0006956. [CrossRef] [PubMed]

23. Caldwell, J.C. How Is Greater Maternal Education Translated into Lower Child Mortality? Health Transit. Rev. 1994, 4, 224-229. Available online: https://www.jstor.org/stable/40652138 (accessed on 25 April 2019).

24. Gakidou, E.; Cowling, K.; Lozano, R.; Murray, C.J.L. Increased educational attainment and its effect on child mortality in 175 countries between 1970 and 2009: A systematic analysis. Lancet 2010, 376, 959-974. [CrossRef]

25. Graetz, N.; Friedman, J.; Osgood-Zimmerman, A.; Burstein, R.; Biehl, M.H.; Shields, C.; Mosser, J.F.; Casey, D.C.; Deshpande, A.; Earl, L.; et al. Mapping local variation in educational attainment across Africa. Nature 2018, 555, 48-53. [CrossRef]

26. Basu, A.M.; Stephenson, R. Low levels of maternal education and the proximate determinants of childhood mortality: A little learning is not a dangerous thing. Soc. Sci. Med. 2005, 60, 2011-2023. [CrossRef]

27. World Health Organization. WHO Housing and Health Guidelines; World Health Organization: Geneva, Switzerland, 2018; Available online: http://apps.who.int/bookorders (accessed on 28 September 2020).

28. Berendes, D.; Leon, J.; Kirby, A.E.; Clennon, J.; Raj, S.; Yakubu, H.; Robb, K.; Kartikeyan, A.; Hemavathy, P.; Gunasekaran, A.; et al. Household sanitation is associated with lower risk of bacterial and protozoal enteric infections, but not viral infections and diarrhoea, in a cohort study in a low-income urban neighbourhood in Vellore, India. Trop. Med. Int. Health 2017, 22, 1119-1129. [CrossRef]

29. McQuade, E.T.R.; Platts-Mills, J.A.; Gratz, J.; Zhang, J.; Moulton, L.H.; Mutasa, K.; Majo, F.D.; Tavengwa, N.; Ntozini, R.; Prendergast, A.J.; et al. Impact of Water Quality, Sanitation, Handwashing, and Nutritional Interventions on Enteric Infections in Rural Zimbabwe: The Sanitation Hygiene Infant Nutrition Efficacy (SHINE) Trial. J. Infect. Dis. 2019. [CrossRef]

30. MAL-ED. The Interactions of Malnutrition and Enteric Infections: Consequences for Child Health and Development. 2015. Available online: http://mal-ed.fnih.org/ (accessed on 25 October 2015).

31. Kotloff, K.; Blackwelder, W.C.; Nasrin, D.; Nataro, J.P.; Farag, T.H.; Van Eijk, A.M.; Adegbola, R.A.; Alonso, P.L.; Breiman, R.F.; Faruque, A.S.G.; et al. The Global Enteric Multicenter Study (GEMS) of Diarrheal Disease in Infants and Young Children in Developing Countries: Epidemiologic and Clinical Methods of the Case/Control Study. Clin. Infect. Dis. 2012, 55, S232-S245. [CrossRef]

32. Humphrey, J.H.; Jones, A.D.; Manges, A.; Mangwadu, G.; Maluccio, J.A.; Mbuya, M.N.N.; Moulton, L.H.; Ntozini, R.; Prendergast, A.J.; The Sanitation Hygiene Infant Nutrition Efficacy (SHINE) Trial Team; et al. The Sanitation Hygiene Infant Nutrition Efficacy (SHINE) Trial: Rationale, Design, and Methods. Clin. Infect. Dis. 2015, 61, S685-S702. [CrossRef] [PubMed]

33. Liu, J.; Gratz, J.; Amour, C.; Nshama, R.; Walongo, T.; Maro, A.; Mduma, E.; Platts-Mills, J.; Boisen, N.; Nataro, J.; et al. Optimization of Quantitative PCR Methods for Enteropathogen Detection. PLoS ONE 2016, 11, e0158199. [CrossRef]

34. World Health Organization. UNICEF. Joint Monitoring Programme (JMP) for Water Supply and Sanitation. 2019. Available online: https://washdata.org/ (accessed on 26 April 2013).

35. Florey, L.; Taylor, C. Using Household Survey Data to Explore the Effects of Improved Housing Conditions on Malaria Infection in Children in Sub-Saharan Africa; ICF International: Rockville, MD, USA, 2016; Available online: https://dhsprogram.com/publications/publication-AS61-Analytical-Studies.cfm (accessed on 30 May 2019).

36. Tickell, K.D.; Sharmin, R.; Deichsel, E.L.; Lamberti, L.M.; Walson, J.L.; Faruque, A.S.G.; Pavlinac, P.B.; Kotloff, K.L.; Chisti, M.J. The effect of acute malnutrition on enteric pathogens, moderate-to-severe diarrhoea, and associated mortality in the Global Enteric Multicenter Study cohort: A post-hoc analysis. Lancet Glob. Health 2020, 8, e215-e224. [CrossRef] 
37. Zou, G.Y. A modified poisson regression approach to prospective studies with binary data. Am. J. Epidemiol. 2004, 159, 702-706. [CrossRef]

38. StataCorp. Stata Statistical Software: Release 16; StataCorp LLC: College Station, TX, USA, 2019.

39. Brown, J.; Cumming, O. Perspective Piece Stool-Based Pathogen Detection Offers Advantages as an Outcome Measure for Water, Sanitation, and Hygiene Trials. Am. J. Trop. Med. Hyg. 2019, 102, 1-2. [CrossRef]

40. Humphrey, J.H.; Mbuya, M.N.N.; Ntozini, R.; Moulton, L.H.; Stoltzfus, R.J.; Tavengwa, N.V.; Mutasa, K.; Majo, F.; Mutasa, B.; Mangwadu, G.; et al. Independent and combined effects of improved water, sanitation, and hygiene, and improved complementary feeding, on child stunting and anaemia in rural Zimbabwe: A cluster-randomised trial. Lancet Glob. Health 2019, 7, e132-e147. [CrossRef]

41. Berendes, D.; Leon, J.S.; Kirby, A.E.; Clennon, J.A.; Raj, S.J.; Yakubu, H.; Robb, K.A.; Kartikeyan, A.; Hemavathy, P.; Gunasekaran, A.; et al. Associations between open drain flooding and pediatric enteric infections in the MAL-ED cohort in a low-income, urban neighborhood in Vellore, India. BMC Public Health 2019, 19, 926. [CrossRef] [PubMed]

42. Qamar, F.N.; Nisar, M.I.; Quadri, F.; Shakoor, S.; Sow, S.; Nasrin, D.; Blackwelder, W.C.; Wu, Y.; Farag, T.; Panchalingham, S.; et al. Aeromonas-Associated Diarrhea in Children under 5 Years: The GEMS Experience. Am. J. Trop. Med. Hyg. 2016, 95, 774-780. [CrossRef]

43. George, C.M.; Perin, J.; Talukder, K.A.; Biswas, S.K.; Oldja, L.; Azmi, I.J.; Faruque, A.G.; Bhuyian, S.I.; Parvin, T.; Sack, R.B.; et al. Fecal Markers of Environmental Enteropathy are Associated with Animal Exposure and Caregiver Hygiene in Bangladesh. Am. J. Trop. Med. Hyg. 2015, 93, 269-275. [CrossRef]

44. Whiley, H.; Akker, B.V.D.; Giglio, S.; Bentham, R. The Role of Environmental Reservoirs in Human Campylobacteriosis. Int. J. Environ. Res. Public Health 2013, 10, 5886-5907. [CrossRef]

45. Marquis, G.S.; Ventura, G.; Gilman, R.H.; Porras, E.; Miranda, E.; Carbajal, L.; Pentafiel, M. Fecal contamination of shanty town toddlers in households with non-corralled poultry, Lima, Peru. Am. J. Public Health 1990, 80, 146-149. [CrossRef]

46. Berrang, M.E.; Northcutt, J.K.; Cason, J.A. Recovery of Campylobacter from Broiler Feces During Extended Storage of Transport Cages. Poult. Sci. 2004, 83, 1213-1217. [CrossRef]

47. Carron, M.; Chang, Y.-M.; Momanyi, K.; Akoko, J.; Kiiru, J.; Bettridge, J.; Chaloner, G.; Rushton, J.; O’Brien, S.; Williams, N.J.; et al. Campylobacter, a zoonotic pathogen of global importance: Prevalence and risk factors in the fast-evolving chicken meat system of Nairobi, Kenya. PLoS Negl. Trop. Dis. 2018, 12, e0006658. [CrossRef]

48. Cardinale, E.; Tall, F.; Guèye, E.; Cisse, M.; Salvat, G. Risk factors for Campylobacter spp. infection in Senegalese broiler-chicken flocks. Prev. Vet. Med. 2004, 64, 15-25. Available online: https://linkinghub. elsevier.com/retrieve/pii/S016758770400087X (accessed on 8 April 2020). [CrossRef] [PubMed]

49. Ngure, F.; Gelli, A.; Becquey, E.; Ganaba, R.; Headey, D.; Huybregts, L.; Pedehombga, A.; Sanou, A.; Traore, A.; Zongo, F.; et al. Exposure to Livestock Feces and Water Quality, Sanitation, and Hygiene (WASH) Conditions among Caregivers and Young Children: Formative Research in Rural Burkina Faso. Am. J. Trop. Med. Hyg. 2019, 100, 998-1004. [CrossRef]

50. Sultana, R.; Nahar, N.; Rimi, N.A.; Azad, S.; Islam, M.S.; Gurley, E.S.; Luby, S.P. Backyard poultry raising in Bangladesh: A valued resource for the villagers and a setting for zoonotic transmission of avian influenza. A qualitative study. Rural. Remote Health 2012, 12, 1927.

51. Penakalapati, G.; Swarthout, J.; Delahoy, M.J.; McAliley, L.; Wodnik, B.; Levy, K.; Freeman, M.C. Exposure to Animal Feces and Human Health: A Systematic Review and Proposed Research Priorities. Environ. Sci. Technol. 2017, 51, 11537-11552. [CrossRef] [PubMed]

52. Psaki, S.R.; Seidman, J.C.; Miller, M.A.; Gottlieb, M.; A Bhutta, Z.; Ahmed, T.; Ahmed, A.S.; Bessong, P.O.; John, S.; Kang, G.; et al. Measuring socioeconomic status in multicountry studies: Results from the eight-country MAL-ED study. Popul. Health Metrics 2014, 12, 8. [CrossRef]

53. Rosenbaum, P.R.; Rubin, D.B. The Central Role of the Propensity Score in Observational Studies for Causal Effects. Biom. JSTOR 1983, 70, 41. [CrossRef]

54. Clasen, T.F.; Alexander, K.T.; Sinclair, D.; Boisson, S.; Peletz, R.; Chang, H.H.; Majorin, F.; Cairncross, S. Interventions to improve water quality for preventing diarrhoea. Cochrane Database Syst. Rev. 2015, 1-201. [CrossRef] [PubMed]

55. Burnett, E.; Jonesteller, C.L.; Tate, J.E.; Yen, C.; Parashar, U.D. Global Impact of Rotavirus Vaccination on Childhood Hospitalizations and Mortality From Diarrhea. J. Infect. Dis. 2017, 215, 1666-1672. [CrossRef] 
56. Colston, J.; Olortegui, M.P.; Zaitchik, B.F.; Yori, P.P.; Kang, G.; Ahmed, T.; Bessong, P.O.; Mduma, E.; Bhutta, Z.A.; Shrestha, P.S.; et al. Pathogen-Specific Impacts of the 2011-2012 La Niña-Associated Floods on Enteric Infections in the MAL-ED Peru Cohort: A Comparative Interrupted Time Series Analysis. Int. J. Environ. Res. Public Health 2020, 17, 487. [CrossRef]

Publisher's Note: MDPI stays neutral with regard to jurisdictional claims in published maps and institutional affiliations.

(C) 2020 by the authors. Licensee MDPI, Basel, Switzerland. This article is an open access article distributed under the terms and conditions of the Creative Commons Attribution (CC BY) license (http://creativecommons.org/licenses/by/4.0/). 